\title{
Interoperability at the core strategic in the Spatial Data Infrastructure of the Service Aerophotogrammetric of the Air Force of Chile.
}

\author{
Joselyn Robledo Ceballos
}

Aerophotogrametric Service, joselyn.robledo@saf.cl

\begin{abstract}
:
The Aerophotogrammetric Service (SAF) of the Chilean Air Force works permanently on the implementation of new methodologies and lines of research, fostering innovation in the field of earth sciences, remote sensing and geospatial information management. The above, with the aim of being at the technical and technological forefront in the country. Proof of this is the implementation of the SAF's Spatial Data Infrastructures, which has as one of its strategic axes the interoperability of geographic information, as it is considered a key factor in the correct exploitation of data, its access, availability and its potential use in decision making.
\end{abstract}

Keywords: SDI, Interoperability, ISO TC/211

\section{Introduction}

Today information and geospatial analysis are key in daily life and in decision making. This means managing a large amount of knowledge (Big Data) that without the concept of interoperability it would be impossible to do. An example of this are the problems that the world has, and that cross borders without respecting jurisdictional lines, such as climate change, threats to national security or flu or pandemics such as that experienced by Covid-19. As already mentioned, trying to solve these problems effectively requires large volumes of data, of known quality, easily accessible and standardized. In addition, the availability of such information must be through a growing variety of technological devices, including personal digital assistants and cell phones.

Given the above, the interoperability of geographic information is a huge challenge for cartographic institutes and corporate, national and regional spatial data infrastructures. This interoperability goes beyond the standardization of data, since these standards must converge in an interoperable technological infrastructure that simplifies reality, hiding the complexity of the information and allowing the exchange and access to quality data for both organizations and users.

The Aerophotogrammetric Service, having large volumes of information and considering its relevant participation in emergency management and what to do with a national scientist, has taken interoperability in the management of its geographic information as a strategic axis and good practices, with the objective end of creating heterogeneous applications that allow planning and joint analysis of available data to collaborate in decision making. Prior to this, the last 5 years have been working hard on the standardization of information in geographical, quality and technological terms, using regulations that promote said interoperability, both from the ISO TC / 211 Technical Committee and the Open Geospatial regulations Consortium (OGC).

Next, this work compiles and analyzes the main achievements in terms of standardization and interoperability of SAF geospatial information through the corporate SDI guidelines.

\section{SAF interoperability model}

As mentioned in the previous section, the SAF interoperability model is based on the implementation of standards (ISO / OGC), standardized quality control (qualitative and quantitative), the cataloging of its data (metadata), the search semantics (ontologies and gazetteer) and the design and creation of interoperable web applications that allow to unify these data and facilitate decision-making. In order to better understand the model implemented in the SAF, the interoperability concept, its dimensions and how these models were adapted to the "Standards-based SAF Geospatial Information Management System" is described below.

\subsection{Definition of interoperability and its levels}

In this section, various definitions of interoperability will be analyzed in order to identify their different trends and their current status. In the literature there are multiple definitions, some oriented to the technological area (databases, software, hardware) and others more related to data (semantics, standards, quality). In the case of geospatial information, a definition that unifies both worlds is necessary.

According to the classic definition found on the web [1] "Interoperability is defined as the ability to exchange and 
share data between two computer systems or components without the intervention of a third system, so that the shared information or data can be used without requiring prior communication". The Police Information Technology Organization (PITO) adds to the definition of interoperability, [2] "the ability to provide or receive services from other systems." The latter is relevant, since through the creation of web services this synergy is generated between providing and receiving data from other organizations, whether or not they are specialized in geospatial information.

The Spanish Center for Ecological Research and Forest Applications (CREAF) defines this concept as follows: [4] "Interoperability seeks that users can exchange information in a simple, transparent, open, effective and universal way." This makes sense, given that all interoperability efforts are focused on the end user of the data and how seemingly something so complex is easy and accessible.

Regarding the normative, the International Standard ISO19101: "Reference Model", proposes as a definition of interoperability: "the capacity of the systems or components to exchange information and to be able to control cooperative processing between applications".

Paul Miller [8], defines interoperability as a "process aimed at ensuring that the procedures, systems and culture of an organization are treated in such a way as to maximize the opportunities for exchange and reuse of information". This definition is one of the most relevant for the SAF model since it includes the importance of interoperability as an organization and at a production level and not as an isolated concept or referred only to technology or regulations.

It is important to mention that interoperability is made up of levels, since its scope is not restricted to a single scope, the Dublin Core glossary indicates that the levels of interoperability are: semantic, structural and syntactic. Other authors have defined more levels, among which we can mention Semantic, Technical, Syntactic, Pragmatic, Organizational, Conceptual, Dynamic, Legal, Social, Schematic or Structural, Intracommunity, HumanPolitical, International, Empirical and Physical. Based on the above, and based on experience, for the SAF model, the most relevant levels are those established in the European Interoperability Framework (EIF), which are technical, semantic and organizational interoperability. These are described below in figure 1 . It should be mentioned that the SAF interoperability model has a lot to do with what is established in EIF since these levels were considered in the RM-ODP model (The ISO Reference Model for Processing Open and Distributed) when designing and implementing the IDE-SAF. EIF defines three types of interoperability:
- Technical interoperability: connection of systems through agreements on norms and standards for the presentation, collection, exchange, transformation and transport of data.

- Semantic interoperability: ensure that the transferred data share the same meaning for linked systems.

- Organizational interoperability: organization of business processes and internal organizational structures, for better data exchange.

\subsection{SAF interoperability model dimensions and lines of work}

The concept of interoperability makes sense when we relate it to a spatial data infrastructure. Among the most accepted definitions of SDI (SDI-Spain) we have that: "A Spatial Data Infrastructure (SDI) is an information system integrated by a set of resources such as catalogs, servers, programs, data, applications and Web pages, dedicated to manage Geographic Information (maps, orthophotos, satellite images, place names, etc.), available on the Internet, that meet a series of interoperability conditions (standards, specifications, protocols, interfaces, etc.), and that allow a user to Using a simple browser, you can use and combine them according to your needs". In this sense, the Aerophotogrammetric Service adopted three of the five dimensions described by EIF (European Interoperability Framework) in the field of interoperability: technical interoperability, semantic interoperability and organizational interoperability. The foregoing, due to its character as Institutional SDI, losing relevance of legal interoperability and integration with the government's public services. The foregoing does not imply that progress will not be made in a parallel manner in these dimensions in the future.

Next, table 1 briefly describes how the dimensions were approached and which were the lines of work in each of them.

\begin{tabular}{|l|l|}
\hline Technical & $\begin{array}{l}\text { Implementation of ISO TC / 211 standards } \\
\text { and OGC standards for: } \\
\text { 1. }\end{array}$ \\
Definition of technical specifications \\
according to ISO 19131 Geographic \\
information \\
specifications.
\end{tabular}




\begin{tabular}{|l|l|}
\hline & $\begin{array}{c}\text { information - Metadata - Part 2: } \\
\text { Extensions for acquisition and } \\
\text { processing. }\end{array}$ \\
$\begin{array}{l}\text { Publication through service and } \\
\text { application websites according to ISO } \\
\text { TC / 211 (theory) and OGC (in } \\
\text { practice) standards. }\end{array}$ \\
\hline $\begin{array}{l}\text { Semantic } \\
\text { Interoperability }\end{array}$ & $\begin{array}{l}\text { Development of a semantic search engine } \\
\text { based on ontologies (RDF) and a gazetteer. }\end{array}$ \\
\hline $\begin{array}{l}\text { Organizational } \\
\text { Interoperability }\end{array}$ & $\begin{array}{l}\text { 1. SDI-SAF strategic and operational } \\
\text { policies. } \\
\text { 2. Determination of actors and roles. }\end{array}$ \\
$\begin{array}{l}\text { 3. Traceability of geospatial information. } \\
\text { 4. Management and quality assurance (ISO } \\
\text { 9001 and ISO 19158: Geographic } \\
\text { information - Quality Assurance of data } \\
\text { supply). }\end{array}$ \\
\hline
\end{tabular}

Table 1. Dimensions of interoperability of the SDI-SAF based on the model of the European Interoperability Framework (EIF).

\subsection{Technical interoperability, SAF model}

In the following model, the SAF technical interoperability scheme based on international standards such as ISO 9001, TC / 211 and OGC is presented. This geospatial information management system was implemented according to the life cycle of a cartographic product (fig.1).

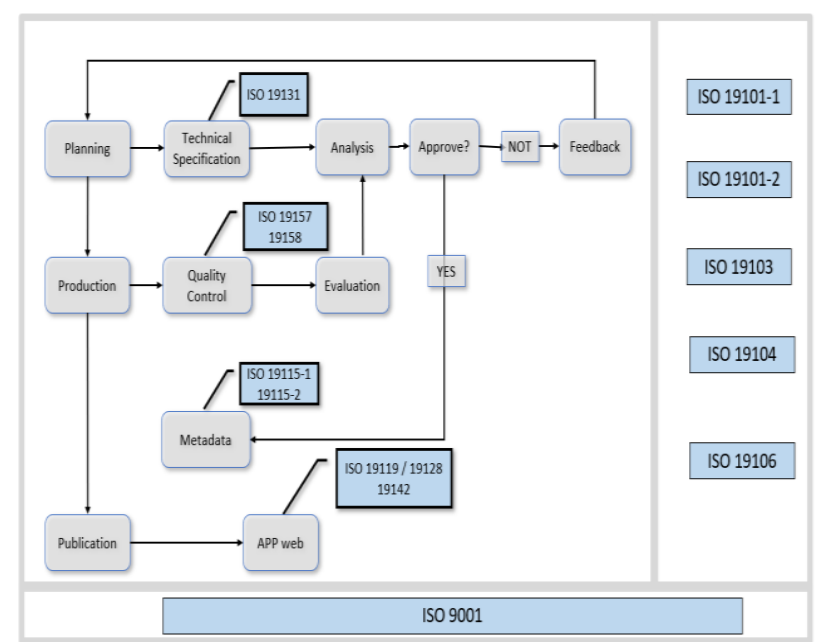

Figure 1: Model of management of the Quality of the Geoespatial information in the SAF.

The implemented model requires a selection and classification of the ISO TC / 211 and OGC standards, according to the product life cycle and identifying normative roles throughout the process, then the selected standards are integrated into the cycle, standardizing the activities in each of the stages.

On the other hand, there are some ISO TC / 211 standards that are related to system management such as ISO 19158: Quality Assurance or regulations that serve as support such as ISO 19101: Reference Model or ISO 19103: Schemes Model UML. To view the SDI -SAF model, see: https://www.researchgate.net/publication/334379234_Mo del_of_Management_of_the_Quality_of_the_Geospatial_
Information_in_the_Aerophotogrametric_Service_of_the _Air_Force_of_Chileed_TC11.

Continuing with technical interoperability, it is worth mentioning that this dimension is the most complex to achieve, since it requires arduous normative and procedural work that can last years and that few organizations are willing to face. The four most relevant guidelines for the technical interoperability dimension in the SAF model are detailed below.

\section{- Technical specifications and design of databases}

ISO 19131: Geographic information - Technical specifications, are fundamental in the product design stage, since they define in a standardized way the client's requirement such as: product use, information levels, quality control (precision, accuracy and tolerances), cataloging and means of delivery. These guidelines are also related to the design of the databases (ISO 19110: Geographic information - Methodology for feature the Cataloging and ISO 19111: Geographic information Referencing by coordinates) which will be the mother structures of the future interoperability of the SAF geospatial databases, since, independent of the client, all comply with a formal structure that allows them to be interrelated and can be cataloged and consumed by web services using the same interoperable protocols.

- Quality of geospatial information and its relevance in the interoperability of SDI-SAF

The quality of the geospatial information is a key factor to achieve interoperability between different sets of spatial data. One of the most studied factors is positional accuracy, since a good positional quality is needed when a set of data of different origin is going to be used, as is the case with SDIs. Different positional behaviors mean the existence of a positional distortion between them, and a barrier to their effective interoperability (Chuch [9] and Col.1998), but the geometric aspect is not the only relevant aspect, there is also the topological and thematic. Studies such as those by MacGwire 1996 and Carmel et al in 2001 and 2006 indicate that thematic quality is highly influenced by positional misalignments. Given the above, for SDI SAF the quality factor is essential and is controlled throughout the entire production process using the methodologies set forth in ISO 19157: Geographic information - Quality Control and ISO 19158: Geographic information - Quality Assurance of data supply, the level of complexity of these Controls vary according to the cartographic product that is being developed.

\section{- Metadata}


Metadata (data on data or data on resources), at present continue to have a secondary nature and are little valued, since they are considered more an expense than an investment, but in the field of SDI and interoperability they are essential, since that efficient access to data in a distributed way through web search engines is only achieved through their metadata. It is a necessary standardization to access large volumes of information in an agile and automated way so that the different systems or organizations that handle geospatial data can interoperate under a common language.

Metadata is used to describe the content, quality, conditions and other characteristics of the data, so that users can access and exploit this information correctly.

The metadata must be standardized, in the case of SAF the standards ISO 19115-1: Geographic information Metadata - Part 1: Fundamentals - Amendment 2 and 19115-2: Geographic information - Metadata - Part 2: Extensions for acquisition and processing.

- Publication of geospatial information

The SDI-SAF has been developing a series of interoperable web services and applications based on ISO TC / 211 and OGC standards, these services use the protocols listed below:

- WFS: Web Feature Service or Vector Feature Service that provides the information related to the feature stored in a vector layer (coverage), which meet the characteristics formulated in the query.

- WMS - Web Map Service or Map Service on the web that produces maps in image format on demand to be viewed by a web browser.

- WCS - Web Coverage Service or Web coverage service (raster data).

- CSW - Web Catalog Service or Catalog Service.

Currently there are applications made up of web services, which collaborate with the Institution in making decisions according to different problems such as:

- Visualization and download of Fasat Charlie images, for the study of camps, through an agreement with the Ministry of Housing and Urbanism (MINVU).

- Applications related to emergencies (Floods, searches: C-130 aircraft in the Beagle Channel, fires, pandemic, etc.).

- Applications that collaborate with the logistics and administrative management of the Institution.

- Applications for availability, visualization and downloading of products and environmental studies such as Las Petras forest or Macul ravine.

- $\quad 3$ D applications for high resolution models.
- Catalog of metadata with more than 7000 metadated products.

These applications are the objective evidence of the levels of interoperability that can be achieved, since they are unified in a single tool independent of the origin of the data and thus provide useful geospatial information in command for decision-making.

\subsection{Semantic Interoperability}

The SDI-SAF Portal, has a semantic search engine prepared by professionals from the University of Concepción, its objective is to have an intelligent search engine to discover the catalog information. This system has the following general characteristics:

- It has a Resource Description Framework (RDF) schema design, which supports the RDF of metadata of SAF geographic information and that links to the SAF product search service through the IDE portal.

- It has a Gazetteer service that allows to associate toponyms (geographical names) to different georeferencing systems, in such a way that it facilitates queries starting indistinctly or continuously from geographic or toponymic criteria.

The semantic search engine allows, searches by metadata keywords and their semantic relations of generalization and synonymy, toponymy and spatial relations established in the RDF scheme. This produces an expanded query that is performed on the RDF and must link the search by metadata to the existing services in the SDI-SAF portal. The architecture is based on free software and on the spatial database that supports the gazetteer. The architecture (Fig. 2) follows the ISO and OGC recommendations of the SDI-SAF.



Figure 2: Architecture of the semantic search engine of the SDI-SAF.

\subsection{Organizational interoperability}

This interoperability is related to the commitment and impact that occurs in the organization with the 
implementation of the SDI, not only in the field of geospatial information management, but also in how to involve the organization with the strategy of the SDI. In the case of theSAF, it is achieved by committing the different areas, documenting the strategic policies that involve the Directorate and the operational policies that involve the technical area.

On the other hand, knowledge management and the vision of innovation are also relevant for the future maintenance of this infrastructure, of its technical and technological improvement, allowing the organization to consider the SDI-SAF and its interoperability model as something every day in the organization.

\section{Conclusion}

Achieving interoperability in an organization is a challenge now and it will continue to be so in the future, until sufficient levels of knowledge, standardization and technology are achieved locally and globally.In spite of this, it is relevant to start interoperating at the SDI level Corporate or cartographic organizations, which is where the data is born, because if this concept is adopted in the genesis of information, interoperability at higher levels of SDIs will be much simpler and faster.

Regulatory efforts are always the most difficult to incorporate, as standards in general are complex and diverse. Based on this, some recommendations later:

- Analyze the production lines and determine their life cycle.

- Determine critical processes related to interoperability dimensions.

- Select the minimum to begin to interoperate within the corporate SDI environment.

- In parallel, determine the technological base with which it has and, based on this, establish the possible levels of interoperability.

- Incorporate into the organization and consider SDI and its interoperability as the standard and normalized way of working.

- Project the technological and regulatory requirements to continue moving towards full interoperability.

At SAF, the challenges around interoperability have been successfully met so far, with today one of the most robust and well-known standards-based management and quality control systems for geospatial information nationally and in the region. Likewise, it has been validated internationally in seminars such as ICC 2017 and ICC 2019 (International Cartographic Conference) and in technical meetings of the ICA (International Cartographic Association). However, given the technological advance and the constant possibilities for innovation, work will continue in this area on a permanent basis, projecting improvements to both the model and the method.

For a more detailed description of these methodologies and of the Management and Control System of Quality of the $\mathrm{SAF}$, it is recommended to consult previous publications of Good Practices of SDI Chile, o international publications such as the "Guide for the evaluation of the positional accuracy of data Spaces" collaboration for the Pan American Institute of History and Geography (PAIGH) of the year 2019, or the international publication ICC2019 / Tokyo: "Geospatial quality management model information in the Aerophotogrammetric Service "

\section{References}

[1] Goodchild M, Egenhofer M, Fegeas R 1997 Interoperating GISs: Report of a specialist meeting held under the auspices of the VARENIUS project. http://www.ncgia.ucsb.edu/Publications/Varenius_Reports/Inter op.pdf.

[2] Tolk A 2003 Beyond Technical InteroperabilityIntroducing a Reference Model for Measures of Merit for Coalition Interoperabiliy, Proceedings of the 8th ICCRTS, Washington, D.C., June 17-19, 2003.

[3] Gómez-Dueñas Laureano-Felipe, Modelos de Interoperabilidad en Bibliotecas Digitales y Repositorios Documentales: Caso Biblioteca Digital Colombiana BDCOL., 2009 (Unpublished) [Report]

[4] Centro de Investigación Ecológica y Aplicación Forestal, Interoperabilidad; https://www.creaf.uab.es/miramon/new_note/esp/notes/C REAF_Interoperabilitat_ESP.pdf

[5] Geospatial Interoperability Return on Investment Study, NASA, abril 2005, http://lasp.colorado.edu/media/projects/egy/files/ROI_Study.pdf

[6] Model of Management of the Quality of the Geospatial Information in the Aerophotogrametric Service, Icc2009 Tokyo, J. Robledo.

[7] Guía para la evaluación de la exactitud posicional de datos espaciales, 2019, IPGH http://publicaciones.ipgh.org/publicaciones-

ocasionales/Guia_Evaluacion_Exactitud_Posicional_ Datos_Espaciales.pdf .

[8] Miller, Paul: "An Introduction to the Resource Description Framework". En: D-Lib Magazine (May 1998) (= MILLER 1998). 